УДК $517.95+537.63+57.04$

\title{
Mathematical Modeling of the Impact Produced by Magnetic Disks on Living Cells
}

\author{
Valery V. Denisenko* \\ Vladimir M. Sadovskii ${ }^{\dagger}$ \\ Institute of Computational Modeling SB RAS \\ Akademgorodok, 50/44, Krasnoyarsk, 660036 \\ Institute of Mathematics and Computer Science \\ Siberian Federal University \\ Svobodny, 79, Krasnoyarsk, 660041 \\ Russia \\ Sergey S. Zamay ${ }^{\ddagger}$ \\ Krasnoyarsk Scientific Center SB RAS \\ Akademgorodok, 50, Krasnoyarsk, 660036 \\ Russia
}

Received 23.05.2016, received in revised form 25.06.2016, accepted 17.07.2016

An analytical solution of the problem on elastic deformation of a membrane allows analyzing deformation of typical living cells under the influence of magnetic disks attached to the cells. Based on the numerical solution of two-dimensional elliptic boundary value problems, the magnetic polarizabilities of the disks are obtained as the functions of magnetic permeability of the disk substance. It is shown that typical thin disks made of iron, nickel and cobalt in the mode far from saturation are magnetized substantially the same as a disk with infinite magnetic permeability. Though the solved problems are stationary, the results are also usable in the analysis of quasi-stationary processes such as low-frequency variation of external magnetic field.

Keywords: elliptic boundary value problem, magnetic polarizability, elastic membrane, living cell. DOI: 10.17516/1997-1397-2016-9-4-432-442.

\section{Introduction}

The recently developed methods of destruction of cancer cells involve mechanical impact induced by microscopic magnetic bodies attached to a cell membrane in different ways [5, 6]. Usually these bodies have shapes of thin disks. The mathematical modeling of the impact consists in the calculation of the torque applied to a disk by the magnetic field and in the estimation of the elastic forces induced by deformation of a cell. This article offers solutions to stationary problems, though the results are applicable to the analysis of quasi-stationary processes, e.g. deformations under external magnetic fields of low frequency. The description of magnetic and mechanical phenomena uses the SI-system.

\footnotetext{
*denisen@icm.krasn.ru

$\dagger$ sadov@icm.krasn.ru

${ }_{\ddagger}^{\ddagger}$ sergey-zamay@yandex.ru

(c) Siberian Federal University. All rights reserved
} 


\section{Problem of magnetostatics}

The mechanical effect of magnetic field on a magnetic disk is defined by the magnetic moment $\mathbf{P}$ of the disk, which is given by integrating magnetization $\mathbf{J}$ over the disk volume

$$
\mathbf{P}=\int \mathbf{J} d \Omega
$$

In the framework of a simplest model [7], the magnetization is linearly related to the magnetic field strength $\mathbf{H}$

$$
\mathbf{J}=\chi \mathbf{H}
$$

where $\chi$ is the magnetic susceptibility, $\mu=1+\chi$ is the relative magnetic permeability.

For ferromagnetic materials, this law is valid at the comparatively low $\mathbf{H}$ with absence of initial magnetization, which automatically holds true in low-frequency variable magnetic field with zero average value. The description of micro- and nano-objects has to use a more advanced micro-magnetic model [7].

The law (1.2) is in accordance with the expression for the magnetic induction $\mathbf{B}$

$$
\mathbf{B}=\mu \mu_{0} \mathbf{H}
$$

where $\mu_{0}$ is the vacuum magnetic permeability. In the weak magnetic fields, cobalt and nickel possess magnetic permeability of round 80 and 400, respectively. Details of properties of ferromagnetics can be found in the monograph [1]. There is no additional notation introduced for the discontinuous function as it is implied that $\mu=1$ outside a body and a prescribed constant $\mu$ inside the body.

The spatial distribution of a magnetic field is found from solving equations of magnetostatics

$$
\begin{aligned}
& \operatorname{div} \mathbf{B}=0, \\
& \operatorname{rot} \mathbf{H}=0,
\end{aligned}
$$

that account for the absence of extraneous currents in our problems. Also $\mathbf{B}$ and $\mathbf{H}$ are related as in (1.3).

Let a ferromagnetic disk be placed in a given uniform magnetic field $\mathbf{H}^{\infty}$. The external field distortion diminishes with the distance from a body; therefore, the required field is to satisfy a boundary condition that is really set not at infinity but at a certain great distance from the center of the disk

$$
\left.\mathbf{H}_{\tau}\right|_{|\mathbf{r}|=\mathbf{R}_{\infty}}=\mathbf{H}_{\tau}^{\infty} .
$$

Here, $\mathbf{r}$ is a point radius-vector; the subscripts $\nu, \tau$ denote the normal and tangential components of vectors.

The second equation in (1.4) allows introducing a potential $V$ such that

$$
\mathbf{H}=-\operatorname{grad} V \text {. }
$$

Then, the magnetostatics problem (1.3)-(1.5) reduces to a Dirichlet problem for one equation

$$
\begin{aligned}
& -\operatorname{div}(\mu \operatorname{grad} V)=0, \\
& \left.V\right|_{|\mathbf{r}|=\mathbf{R}_{\infty}}=-\mathbf{r} \mathbf{H}^{\infty} .
\end{aligned}
$$

Since $\mu$ has strictly positive and limited values (in our problems, $1 \leqslant \mu \leqslant 1000$ ), the equation is elliptical, and the boundary value problem (1.7) has a unique solution.

It is natural to use the axial symmetry of the disk to reduce the $3 \mathrm{D}$ problem (1.7) to a twodimensional problem. To this effect, a cylindrical coordinate system $r, \varphi, z$ is introduced, with 
the $z$ axis directed along the disk axis and the $x$ axis of the related Cartesian coordinates placed in a half-plane where $\mathbf{H}^{\infty}$ is. This is the half-plane $\varphi=0$. In case that $\mathbf{H}^{\infty}$ is parallel to the $z$ axis, the $x$ axis is chosen arbitrarily. Denote the angle between the $z$ axis and $\mathbf{H}^{\infty}$ by $\theta_{\infty}$. Then

$$
\begin{gathered}
H_{z}^{\infty}=H^{\infty} \cos \theta_{\infty}, \\
H_{x}^{\infty}=H^{\infty} \sin \theta_{\infty}, \\
H_{y}^{\infty}=0 .
\end{gathered}
$$

By the linearity of the problem (1.7), it is possible to consider independently a pair of problems with a single non-zero component $H_{z}^{\infty}$ or $H_{x}^{\infty}$ and, then, to sum up the solutions. The expediency of this separation is governed by the difference of two-dimensional formulations of these two problems.

\section{Axisymmetrical field}

When the given external field is oriented along the axis of the disk, the required field possesses the axial symmetry and the potential $V$ is independent of the angle $\varphi$. Therefore it is possible to write down the problem (1.7) in the form

$$
\begin{gathered}
-\frac{\partial}{\partial r}\left(r \mu \frac{\partial V}{\partial r}\right)-\frac{\partial}{\partial z}\left(r \mu \frac{\partial V}{\partial z}\right)=0 . \\
\left.V\right|_{r^{2}+z^{2}=R_{\infty}^{2}}=-z H_{z}^{\infty}
\end{gathered}
$$

where the equation is already multiplied by $r$.

The parameter $r \mu$, which is a coefficient in this equation, vanishes in the axis $r=0$. For the problem solution to agree with the smooth solution of the original three-dimensional problem, it is required that

$$
\left.\frac{\partial V}{\partial r}\right|_{r=0}=0 .
$$

In fact, the condition is implemented at a small distance $R_{0}$ from the $z$ axis as

$$
-\left.r \mu \frac{\partial V}{\partial r}\right|_{r=R_{0}}=0
$$

This is a no-flux condition for a flux with a density $-r \mu \operatorname{grad} V$ and this is a natural condition for the equation (2.1).

By antisymmetry relative to the half-line $z=0$, the problem is solvable in a quarter of the plane $z>0, r>R_{0}$ with one more boundary condition

$$
\left.V\right|_{z=0}=0 \text {. }
$$

The solution of the mixed boundary value problem (2.1)-(2.3) exists and is unique. In view of the same properties of a 3D problem, just this solution is of interest.

The problem has been solved numerically. The used multigrid finite element method is represented in the monograph [2] by the first author of the present article. Some applications of the method are considered in the paper [3].

Fig. 1 shows the resultant distribution of the magnetic field for a nickel disk with a radius $R=250 \mathrm{~nm}$ and a thickness $h=60 \mathrm{~nm}$. Furthermore, the figure depicts equipotentials and magnetic field lines, i.e. the lines where the vector $\mathbf{B}$ is tangential at each point. 
The magnetic moment of the disk, solely the component $P_{z} \neq 0$ due to symmetry, after the problem solving, can be found by integrating over the disk volume (1.1)

$$
P_{z}=\int_{0}^{2 \pi} \int_{-h / 2}^{h / 2} \int_{0}^{R} J_{z} d \varphi d z r d r
$$

The integration process accounts for $(1.2,1.6)$ and is simplified owing to the axial symmetry and antisymmetry relative to the plane $z=0$ :

$$
P_{z}=-4 \pi \chi \int_{0}^{R}\left(\int_{0}^{h / 2} \frac{\partial V}{\partial z} d z\right) r d r=-4 \pi(\mu-1) \int_{0}^{R} V(r, h / 2) r d r .
$$

From the problem linearity,

$$
P_{z}=\alpha \Omega H_{z}^{\infty},
$$

where the coefficient $\alpha \Omega$ is the magnetic polarizability of a body as a whole, $\Omega$ is the body volume. Assuming homogeneity of substance, the parameter $\alpha$ is only governed by the body configuration. The value of $\alpha$ is the basic required value.

The representative values of $\alpha$ are obtainable in the framework of a one-dimensional model based on the smallness of the disk thickness relative to the disk radius $h<<R$.

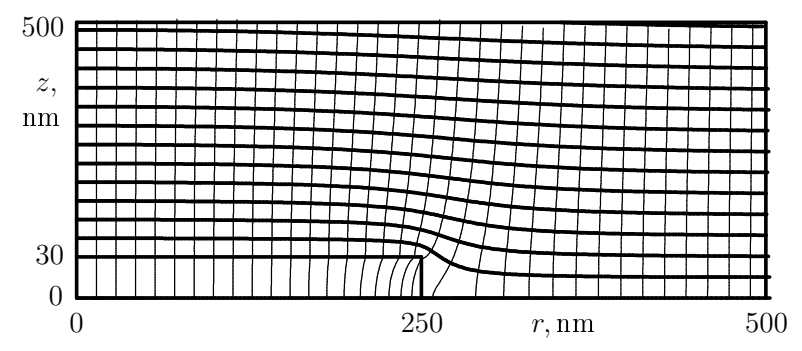

Fig. 1. Distribution of axisymmetrical magnetic field inside and outside a nickel disk, $\mu=400$. The solid lines are equipotentials with a step $H^{\infty} h / 2$, the fine lines are the magnetic field lines. The rectangle $250 * 30 \mathrm{~nm}$ is a quarter of the disk cross section

In this case, the first equation in (1.4) means the approximate constancy $B_{z}=B_{z}^{\infty}$. Accordingly,

are constant inside the disk.

$$
H_{z}=H_{z}^{\infty} / \mu, \quad J_{z}=\frac{\mu-1}{\mu} H_{z}^{\infty}
$$

The magnetic moment of the whole disk is found from multiplication by volume, i.e. the one-dimensional approximation yields

$$
\alpha^{(1)}=1-1 / \mu \text {. }
$$

The function $\alpha^{(1)}(\mu)$ is plotted in Fig. 2 as a fine curve. The solid curve shows the numerical solution of the series of the problems $(2.1)-(2.3)$ with the different values of $\mu$. It is seen in the figure that the disks are sufficiently thin for the one-dimensional model to have error under $40 \%$ for all ferromagnetic materials. The true value of $\alpha$ is higher than in the one-dimensional model since the field is higher at the disk edge than at the disk center adequately described by the one-dimensional model. This is clearly seen in Fig. 1. The basic result is the equivalence of all magnetics with $\mu>10$ subject to the limit accuracy of $10 \%$.

It is worthy of mentioning that when $\mu \rightarrow \infty$, the problem (2.1)-(2.3) is essentially simplified as $V \equiv 0$ inside the disk. So, it is sufficient to solve the problem for a Laplace equation outside the disk with the complementary Dirichlet condition that $V=0$ at the disk boundary. 

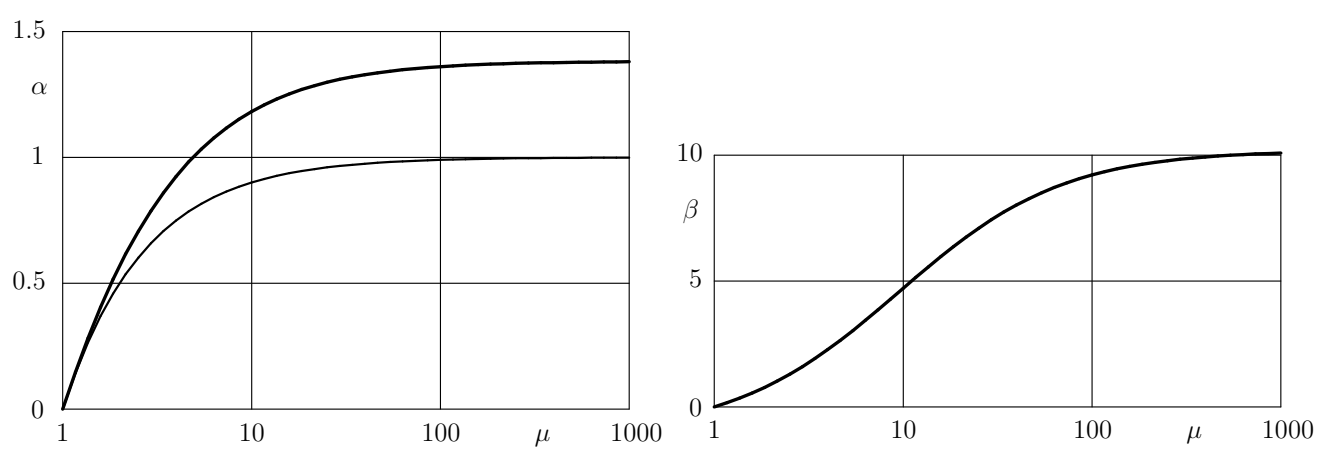

Fig. 2. The solid lines illustrate calculations of the magnetic polarizabilities of the disk, which are divided by the volume of the disk, $\alpha=P_{z} /\left(\Omega H_{z}^{\infty}\right)$ and $\beta=P_{y} /\left(\Omega H_{y}^{\infty}\right)$, as against the disk substance permeability $\mu$. The fine line shows the analytical solution (2.5) of the one-dimensional problem

\section{Magnetization of a disk by a field normal to its axis}

When the external magnetic field is parallel to the disk plane in the boundary value problem (1.7) the function set in the boundary condition is given by

$$
\left.V\right|_{r^{2}+z^{2}=R_{\infty}^{2}}=-r H_{x}^{\infty} \cos \varphi .
$$

The magnetic permeability $\mu$ is independent of the angle $\varphi$ due to axial symmetry of the disk. These two circumstances enable finding the solution in the form

$$
V(r, z) \cos \varphi .
$$

Since the original problem (1.7) has a unique solution, the found solution in the form of (3.1) is the required solution. As the axis has no peculiarities, it is required that $V(r, z)$ vanishes

$$
\left.V\right|_{r=0}=0 .
$$

As it is, the condition is implemented at a small distance $R_{0}$ from the $z$ axis as

$$
\left.V\right|_{r=R_{0}}=0 \text {. }
$$

The problem (1.7), (3.2) takes on form

$$
\begin{gathered}
-\frac{1}{r} \frac{\partial}{\partial r}\left(r \mu \frac{\partial V}{\partial r}\right)-\frac{\partial}{\partial z}\left(\mu \frac{\partial V}{\partial z}\right)+\frac{1}{r^{2}} V=0, \\
\left.V\right|_{r^{2}+z^{2}=R_{\infty}^{2}}=-r H_{x}^{\infty},\left.\quad V\right|_{r=R_{0}}=0 .
\end{gathered}
$$

The equation (3.3) omits the common multiplier $\cos \varphi$ since for the fulfillment of the equation at all values of $\varphi$ is equivalent to zero multiplier in front of $\cos \varphi$.

In view of symmetry of the problem (3.1) relative to the plane $z=0$ the problem (3.3) is solvable in a quarter of the plane with the additional boundary condition

$$
\left.\frac{\partial V}{\partial z}\right|_{z=0}=0 .
$$

The problem $(3.3,3.4)$ has been solved numerically. Similarly to the previous Section, the boundary conditions (3.3) hold true for a relatively small cylinder $r=R_{0}$ and for a large sphere $r^{2}+z^{2}=R_{\infty}^{2}$ with the parameters $R_{0}, R_{\infty}$ selected from test calculations. 
Fig. 3a demonstrates the resultant distribution of the potential $V(r, z)$ and Fig. 3b displays the distribution of this potential over the plane $z=0$ in accordance with (3.1). The field is much weaker inside of the disk, and the equipotentials are therefore plotted with a step 50 times less.
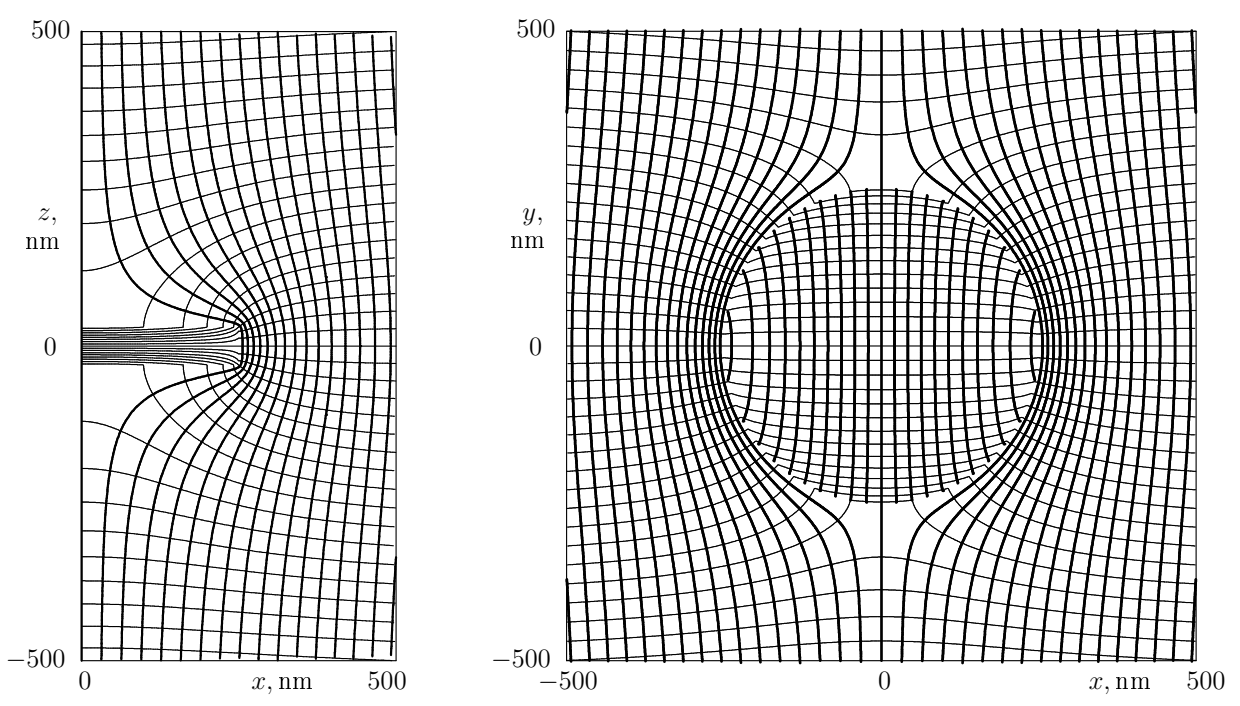

Fig. 3. Distribution of magnetic field inside and outside a nickel disk with the orientation in parallel to the external field. The half-plane $y=0, x>0$ is left panel. The plane $z=0$ is right panel. The fine lines are the magnetic field lines; the solid lines are the equipotentials with the same step $H^{\infty} h / 2$ as in Fig. 1. The equipotentials inside of the disk are plotted at 50 times smaller step

By definition, the tangent direction to a magnetic field line shows the orientation of $\mathbf{B}$. Considering that div $\mathbf{B}$ equals zero in view of (1.4), such lines posses one more essential property namely conservation of flux in a magnetic field tube. In two-dimensional problems, this means that $|\mathbf{B}|$ is inversely proportional to spacing of neighbor field lines, which makes the figures even more informative.

By symmetry, the magnetic moment of the disk has the only $x$-component calculated, in accordance with (1.1), by integration of the magnetization $J_{x}$. On the strength of $(1.2),(1.6),(3.1)$

$$
J_{x}=-\chi \frac{\partial(V \cos \varphi)}{\partial x}=-(\mu-1)\left(\frac{\partial V}{\partial r} \cos ^{2} \varphi+\frac{V}{r} \sin ^{2} \varphi\right) .
$$

The integral (1.1) takes on form

$$
P_{x}=-2 \pi(\mu-1) \int_{0}^{h / 2} d z \int_{0}^{R}\left(\frac{\partial V}{\partial r}+\frac{V}{r}\right) r d r
$$

where the integration with respect to $\varphi$ has already been performed. Let the element of integration be transformed so that to allow analytical integration:

$$
\int_{0}^{R} \frac{\partial(r V)}{\partial r} d r=\left.r V\right|_{0} ^{R}=R V(R, z)
$$

Thus, from (3.5), obtain

$$
P_{x}=-2 \pi R(\mu-1) \int_{0}^{h / 2} V(R, z) d z
$$


Since the problem is linear at the fixed $\mu$ and the disk geometry, the parameter $\beta$ is constant in the formula

$$
P_{x}=\beta \Omega H_{x}^{\infty} .
$$

The relationship of $\beta$ and $\mu$ is illustrated in the right panel in Fig. 2. The curve is plotted based on the solution to the series of problems at different values of $\mu$.

The first basic result is the equivalence of all magnetic materials with $\mu>70$ within an accuracy limited to $10 \%$.

The second important, though expected, result ensues from the comparison of the right and left panels of Fig. 2: magnetization is much higher in the plane of the disk than along its axis, $\beta / \alpha>6.6$ for all $\mu>70$ of interest. Specifically for nickel with $\mu \approx 400$, we have $\alpha \approx 1.37, \beta \approx 9.5, \beta / \alpha \approx 7$.

The problem (3.3) is essentially simplified when $\mu \longrightarrow \infty$ for $V \equiv 0$ inside of the disk. For this reason, it is sufficient to solve the problem for a Laplace equation outside the disk with the complementary Dirichlet condition $V=0$ at the disk boundary.

\section{Motion of disk}

When the external magnetic field is parallel or perpendicular to the disk axis, the generated magnetic moment of the disk is parallel to the field. Using (1.8), (2.4), (3.6) allows writing down

$$
\begin{gathered}
P_{z}=\alpha \Omega H^{\infty} \cos \theta_{\infty}, \\
P_{x}=\beta \Omega H^{\infty} \sin \theta_{\infty}, \\
P_{y}=0 .
\end{gathered}
$$

Such magnetic dipole experiences the torque $\mathbf{N}=\left[\mathbf{P} \times \mathbf{B}^{\infty}\right]$, i.e.

$$
N_{y}=-\Omega \mu_{0}\left(H^{\infty}\right)^{2}(\beta-\alpha) \sin \theta_{\infty} \cos \theta_{\infty}, \quad N_{x}=0, N_{z}=0 .
$$

Naturally, when $\theta_{\infty}=0$ or $\theta_{\infty}=\pi / 2$, the torque vanishes. These positions of the disk are equilibrium but only the second position, when $\theta_{\infty}=\pi / 2$ and the field is in the plane of the disk, is stable.

In case of the arbitrary initial orientation of the disk axis, in the time-independent magnetic field, the disk will oscillate about the equilibrium position. Under loss of energy due to friction, the disk plane will become parallel to the field. In a rotary field in a certain plane, the disk will be positioned in parallel to this plane. When the disk is glued to an elastic surface rather than freely float in fluid, the described motions will complicate. In such mechanical problem, the role of the magnetic field reduces to the generation of the torque (4.2).

\section{Mechanical effect of magnetic disks on cells}

Let a disk with a radius $R$ be completely glued to a membrane and rotated at an angle $\theta$ off the membrane plane. Under concern is a phenomenon having the scale much smaller than a cell radius $R_{c}$, and the curvature of the membrane is neglected therefore. The membrane is assumed to be thin and is supposed to be fixed at a circle with a large radius $R_{\infty}$ and with the center at the same point with the disk center. The formulation involves the polar coordinates $r, \varphi$ of the points in the plane of the undeformed membrane and the related Cartesian coordinates $x, y, z$.

The estimation of elastic forces generated under rotation of the disk assumes approximately that the elastic forces are governed by deformation of the membrane. By the data from [4], membranes of cells of mammals are stretched in the normal condition so that tension is of the 
order of $\sigma=10^{-5} \mathrm{~N} / \mathrm{m}$. The tension of the membrane is compensated by the pressure inside the cells, and the pressure value is higher by $\Delta P=2 \sigma / R_{c}$ than the ambient pressure. In a cell with $R_{c}=10 \mu \mathrm{m}, \Delta P \approx 2 \mathrm{~Pa}$.

The displacement of points along $\varphi$ and $r$ under low straining has higher order of infinitesimals than the displacements in the perpendicular direction to the membrane. Let the latter be denoted by $w(r, \varphi)$. Thus, the displacement of the membrane points is described by one function $w(r, \varphi)$. The inclination of the circle with a radius $R$ by a small angle $\theta$ and the zero rise at a circle $r=R_{\infty}$ yields the boundary conditions

$$
\begin{gathered}
w(R, \varphi)=R \theta \cos \varphi \\
w\left(R_{\infty}, \varphi\right)=0 .
\end{gathered}
$$

The membrane takes a shape such that the elastic energy $I$ is minimum. In accordance with [8]

$$
I=\pi \sigma \int_{R}^{R_{\infty}}\left(\left(\frac{\partial w}{\partial r}\right)^{2}+\left(\frac{1}{r} \frac{\partial w}{\partial \varphi}\right)^{2}\right) r d r
$$

The condition for $I$ of a uniform membrane to be minimal is the fulfilled equation below

$$
\frac{1}{r} \frac{\partial}{\partial r}\left(r \frac{\partial w}{\partial r}\right)+\frac{1}{r^{2}} \frac{\partial^{2} w}{\partial \varphi^{2}}=0 .
$$

The solution is tried by the method of separation of variables in the form of $w(r, \varphi)=$ $f(r) \cos \varphi$, where the dependence on the angle $\varphi$ was at once found from the first boundary condition (5.1). For the function $f(r)$, the equation (5.2) produces an ordinary differential equation

$$
\frac{1}{r} \frac{d}{d r}\left(r \frac{d f(r)}{d r}\right)-\frac{1}{r^{2}} f(r)=0
$$

Its general solution

$$
f(r)=A / r+B r
$$

where $A, B$ are arbitrary constants. The values of the constants are found from the boundary conditions (5.1)

$$
A=\theta R^{2} /\left(1-\left(R / R_{\infty}\right)^{2}\right), \quad B=-\theta\left(R / R_{\infty}\right)^{2} /\left(1-\left(R / R_{\infty}\right)^{2}\right) .
$$

The function $f(r)$ coincident with the two-dimensional solution $w(r, \varphi)$ in the $x$ axis is shown at $\theta=25^{\circ}$ in Fig. 4 .

The membrane is inclined at each point relative to the plane, in particular by an angle $\gamma$ in the radial direction

$$
\operatorname{tg}(\gamma(r, \varphi))=\frac{\partial w(r, \varphi)}{\partial r}=\frac{d f(r)}{d r} \cos \varphi=\left(-\frac{A}{r^{2}}+B\right) \cos \varphi .
$$

At a rigid circle boundary, it is possible to simplify the formula due to smallness of the angle $\gamma$ when $\operatorname{tg} \gamma \approx \gamma \approx \sin \gamma$. Then $\gamma \approx \gamma_{0} \cos \varphi$, where $\gamma_{0} \approx \theta\left(1+\left(R / R_{\infty}\right)^{2}\right) /\left(1-\left(R / R_{\infty}\right)^{2}\right)$.

From symmetry, the forces at the circle boundary produce zero $x-, z-$ components of the torque. Let us find the $y$-component. Under the assumed low straining, the tension of the membrane remains, within small limits, equal to the preset value $\sigma$, accordingly, the membrane section at the arc pulls the circle with a force $d F=\sigma R d \varphi$ in the direction that is tangential to the membrane and is normal to the circle. This direction is approximately set 
by a vector $\mathbf{e} \approx\left(\cos \varphi, \sin \varphi,-\gamma_{0} \cos \varphi\right)$. The vector product of the force point radius-vector $\mathbf{r} \approx(R \cos \varphi, R \sin \varphi, R \theta \cos \varphi)$ and the force gives

$$
d N_{y}=[\mathbf{r} \times \mathbf{e} d F]_{y}=\sigma R^{2}\left(\theta+\gamma_{0}\right) \cos ^{2} \varphi d \varphi .
$$

The integration over the whole circle yields the $y$-component of the torque

$$
N_{y} \approx \sigma \pi R^{2}\left(\theta+\gamma_{0}\right) / 2 \approx \theta \sigma \pi R^{2} /\left(1-\left(R / R_{\infty}\right)^{2}\right) .
$$

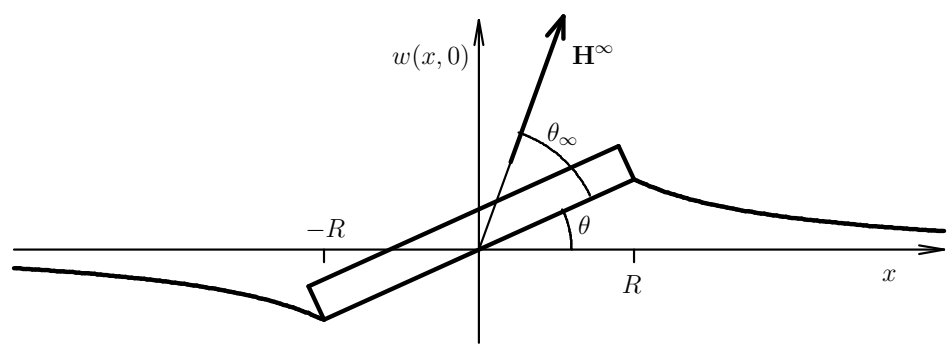

Fig. 4. Deformation of membrane at the disk rotation by $\theta=25^{\circ}$. The rectangle is the disk cross section

This expression includes the parameter $R_{\infty}$, the value of which is only determined by the inequalities $R_{c} \gg R_{\infty} \gg R$. For the employable disks having $R<0.5 \mu \mathrm{m}$, at a cell radius $R_{c}>10 \mu \mathrm{m}$, it is acceptable upon the average that $R_{\infty}=\sqrt{R_{c} R} \approx 2 \mu \mathrm{m}$. Then, it follows from (5.3) with an error of round $4 \%$ that

$$
N_{y} \approx \theta \sigma \pi R^{2}
$$

The found torque of elastic forces neutralizes the torque (4.2) that rotates the magnetized disk in the magnetic field. This equality, with the disk volume $\Omega$ written in terms of the disk radius $R$ and height $h$, yields the disk rotation angle

$$
\theta \approx-0.5 h \mu_{0}\left(H^{\infty}\right)^{2}(\beta-\alpha) \sin \left(2 \theta_{\infty}\right) / \sigma .
$$

The appropriate calculations were performed for a nickel disk with a radius $R=250 \mathrm{~nm}$ and a height $h=60 \mathrm{~nm}$ in external magnetic field with a strength $H^{\infty}=12 \mathrm{kA} / \mathrm{m}=150$ Oe. Nickel in weak fields has relative magnetic permeability $\mu \approx 400$. In Secs. 2 and 3 , the magnetic polarizabilities of the disk were found relative to its volume, $\alpha \approx 1.37$ and $\beta \approx 9.5$. The specific tension of the membrane is $\sigma=10^{-5} \mathrm{~N} / \mathrm{m}$ [4]. Placing these values in (5.5) results in the maximum disk rotation $\theta \approx 25^{\circ}$. The maximum angle of rotation comes when the angle between the external magnetic field and the disk plane is $\theta_{\infty}=45^{\circ}$.

\section{Extension of the application area of the results}

This study offers solutions to stationary problems; at the same time, the results are applicable to analyzing quasi-stationary processes, for instance, low-frequency variations of external magnetic field.

It is safe to mention that the disk rotation angle (5.5) comes only for magnetic disks oriented relative to the magnetic field direction so that $\theta_{\infty}=45^{\circ}$. Inasmuch as particles have arbitrary orientations when attach to cells, the mentioned values of the parameters should be reduced a few times for assessing average influence on a cell. 
In some experiments, for instance, [6], the magnetic field $\mathbf{H}^{\infty}$ varies in time. The involved frequency equals $50 \mathrm{~Hz}$. It is readily assessed that at such low frequencies, both viscosity of fluid and inertia of a disk and surrounding fluid involved in motion are neglectable, and it is assumable that the process is quasi-stationary. The resultant limit values of the disk rotation angle (5.5) are achievable when the magnetic field has the maximum strength, which occurs two times within a period. When the field acquires opposite direction, the magnetic moment of the disk changes the direction as well. For this reason, equal to their vector product, the torque (4.2), which rotates the magnetized disk in the magnetic field, keeps the sign. Accordingly, during the two half-periods, the disk rotates in the same direction as the field gets stronger and recurs free position when the field becomes weaker. Therefore, in $0.01 \mathrm{~s}$, the disk rotates at a maximum angle and resets the initial position conformable with an undeformed membrane.

The presented model assumes linear relation between magnetization of a substance and the magnetic field strength (1.2), and this assumption is approximately valid for ferromagnetic materials in weak fields. The description of micro- and nano-objects requires a more complicated micro-magnetic model [7]. The detailed distributions of magnetization intensity become more complex in this case. For instance, thin micro-disks have circumferencial magnetization nonvanishing as the external magnetic field $\mathbf{H}^{\infty}$ tends to zero. The results obtained in Secs. 2 and 3 in this study are inapplicable to micro- and nano-disks. Nevertheless, the findings on micro-magnetic modeling from [5] mean that the magnetic moment of a micro-disk as a whole is adequately proportional to the strength of the field when it is less than a saturation field and is parallel to the plane of the disk. Moreover, these data can in rough way be written in a (4.1)-conformable form $P_{x}=\beta \Omega H_{x}^{\infty}$ with $\beta \approx 20$. Our calculations for disks used in [5] yield a similar value of $\beta$ at representative values of magnetic permeability $\mu$ from one to eight thousand for permalloy $\mathrm{Fe}_{20} \mathrm{Ni}_{80}$ in macroscopic bodies. These largely scattered values of $\mu$, resultant from different manufacture technologies, have nearly no effect on $\beta$ that is the same at high $\mu$ and at $\mu=\infty$. For disks with a twice as little diameter, the property of magnetic polarizability is illustrated in Fig. 2.

It is possible that for a field with arbitrary orientation relative to a disk, the magnetization intensity and field relation may have the form close to (4.1) for micro-disks despite non-linearity of the micro-magnetic model. Then, the expression (5.5) is valid for the disk rotation angle. Otherwise, at different magnetization intensities, it is only possible to use the estimate of the membrane response to rotation of the glued disk, (5.4), obtained in Sec. 5 in the framework of the theory of elastic thin shells.

\section{Conclusion}

In that way, we have calculated deformations of typical living cells under the influence of magnetic disks glued to these cells.

The relations between the magnetic polarizability of disks and the magnetic permeability of a disk substance have been obtained with the assumption that the magnetic permeability of a substance equals a given constant. This approximation is valid for modes far from saturation, i.e. for weak fields.

This study shows that representative thin disks made of such ferromagnetic materials as iron, nickel and cobalt magnetize in the same way as at infinite magnetic permeability $\mu=\infty$. It is much simpler to solve problems with $\mu=\infty$ since they reduce to the boundary value problems for a Laplace equation outside a domain occupied by a magnetic material.

The article illustrates applicability of the results when initial modeling assumptions are only partly valid.

This study has been supported by the Russian Science Foundation (project 14-15-00805). 


\title{
References
}

[1] R.M.Bozort, Ferromagnetism, Princeton, New Jersey, D. Van Nostrand Company Inc., 1951.

[2] V.V.Denisenko, Energy Methods for Elliptic Equations with Asymmetric Coefficients, Novosibirsk, Publ. house of the SB RAS, 1995 (in Russian).

[3] V.V.Denisenko, Energy Method for Mathematical Modeling of Heat Transfer in 2-D Flow, Journal of Siberian Federal University. Mathematics and Physics, 7(2014), no. 4, 417-428.

[4] N.C.Gauthier, T.A.Masters, M.P.Sheetz, Mechanical feedback between membrane tension and dynamics, Trends in Cell Biology, 22(2012), no. 10, 527-535.

[5] D.-H.Kim, E.A.Rozhkova, I.V.Ulasov, S.D.Bader, T.Rajh, M.S.Lesniak, and V.Novosad, Biofunctionalized Magnetic Vortex Microdisks for Targeted Cancer Cell Destruction, Nature Material, 9(2010), no. 2, 16-17.

[6] P.D.Kim et al., The anti-tumor effect of magnetic nanodisks and DNA aptamer conjugates, Doklady Biochemistry and Biophysics, 466(2016), 66-69.

[7] L.D.Landau, and E.M.Lifshitz, Electrodynamics of Continuous Media (Volume 8 of A Course of Theoretical Physics), Pergamon Press, 1960.

[8] K.Washizu, Variational Methods in Elasticity and Plasticity, Oxford-New York, Pergamon Press, 1982.

\section{Математическое моделирование воздействия магнитных дисков на живые клетки}

\author{
Валерий В. Денисенко \\ Владимир М. Садовский, \\ Институт вычислительного моделирования СО РАН \\ Академгородок, 50/44, Красноярск, 660036 \\ Институт математики и фундаментальной информатики \\ Сибирский федеральный университет \\ Свободный, 79, Красноярск, 660041 \\ Россия \\ Сергей С. Замай \\ Красноярский научный центр СО РАН \\ Академгородок, 50, Красноярск, 660036
}

Россия

\begin{abstract}
Построено аналитическое решение задачи о деформачии упругой мембраны, которое позволяет анализировать деформации типичных живых клеток под воздействием прикрепленных $к$ клеткам магнитных дисков. На основе численного решения двумерных эллиптических краевых задач получены зависимости магнитных поляризуемостей дисков от магнитной поницаемости их вещества. Показано, что типичные тонкие диски, изготовленные из железа, никеля, кобальта, ө режимах, далеких от насыщения, намагничиваются практически так же, как при бесконечной магнитной проницаемости. В работе решены стационарные задачи, однако полученные результаты могут быть использованы и при анализе квазистационарных процессов, происходящих, например, при изменении внешнего магнитного поля с небольшой частотой.
\end{abstract}

Ключевые слова: эллиптическая краевая задача, магнитная поляризуемость, упругал мембрана, живая клетка. 